\title{
Verifying Non-Abelian Statistics by Numerical Braiding Majorana Fermions
}

\author{
Qiu-Bo Cheng ${ }^{\mathrm{a}, 1}$, Jing He ${ }^{\mathrm{b}, 1}$, Su-Peng Kou ${ }^{\mathrm{a}, *}$ \\ ${ }^{a}$ Department of Physics, Beijing Normal University, Beijing, 100875, P. R. China \\ ${ }^{b}$ Department of Physics, Hebei Normal University, Hebei, 050024, P. R. China
}

\begin{abstract}
Recently, Majorana fermions have attracted intensive attention because of their possible non-Abelian statistics and potential applications in topological quantum computation. This paper describes an approach to verify the non-Abelian statistics of Majorana fermions in topological superconductors. From the relationship between the braiding operator of Majorana fermions and that of Bogoliubov-de Gennes states, we determine that Majorana fermions in one-dimensional and two-dimensional topological superconductors both obey non-Abelian statistics.
\end{abstract}

Keywords: Majorana fermions, Topological superconductors

\section{Introduction}

Majorana fermions (MFs) $\gamma_{i}$ are their own antiparticles and constitute 'half' of the ordinary fermions[1, 2, 3] that satisfy $\gamma_{i}=\gamma_{i}^{\dagger}$ and $\left\{\gamma_{i}, \gamma_{j}\right\}=2 \delta_{i j}$. Although it is still unclear whether MFs exist in nature as "elementary building blocks", they may appear as Majorana bound states (MBSs) in certain condensed matter systems [4, 5, 6, 7, 8, 9, 10, 11, 12, 13, 14, 15, 16, 17, 18]. Recently, due to their potential applications in topological quantum computation (TQC)[19, 20, 21, 22, 23], the search for exotic states supporting MFs has attracted increasing interest in condensed matter physics. One possible example of such quantum exotic states is the $p_{x}+i p_{y}$ topological superconductor (SC) state in two dimensions. This state supports MBSs at quantized vortices ( $\pi$-fluxes) $[20,24,25,26]$. Another creative proposal is the interface of a $s$-wave SC and a topological insulator due to the proximity effect[5], in which quantized vortices may also trap MBSs. In addition, a new type of MBSs proposed by Kitaev occurs in a one-dimensional (1D) electronic nanowire proximity-coupled to a bulk superconductor[4]. For this case, two unpaired Majorana zero modes appear at the two ends of the nanowire. In Ref.[27], MBSs are also observed to be trapped by line defects of p-wave superconductors on a honeycomb lattice.

In Ref.[24], the authors reported that the MFs trapped by vortices in $p_{x}+i p_{y}$-wave topological SC obey non-Abelian statistics[28, 29]. After exchanging two MFs in the 2D topological SC, the braiding operation can be represented by $\gamma_{1} \rightarrow \gamma_{2}$, $\gamma_{2} \rightarrow-\gamma_{1}[25]$. For the MFs at the ends of line-defect in SCs, the braiding operations were exhibited to be along T-junction paths[11]. Based on the arguments in Ref.[11], the line-defectinduced MFs were found to also obey non-Abelian statistics. As a result, the MFs in different models (both vortex-induced MFs and the line-defect-induced MFs) were believed to obey

\footnotetext{
${ }^{*}$ Corresponding author; Email address: spkou @ bnu.edu.cn

${ }^{1}$ These authors contributed equally to this paper.
}

the same type of non-Abelian statistics. In this paper, we will introduce a numerical approach to verify the statistics of the MFs. The results will help to elucidate the properties of topological SCs.

\section{Methods}

In certain topological SCs, MFs with zero energy (the Majorana zero modes) may emerge around defects (for example, the quantized vortex or the 1D nano-wire). In general, the function $u(x)$ of an emergent MF can be obtained by solving the Bogoliubov-de Gennes (BdG) equation numerically. The corresponding Majorana operator is defined by $\gamma=$ $\int d x\left[u(x) c^{\dagger}(x)+u^{*}(x) c(x)\right]\left(\gamma^{\dagger}=\gamma\right)$. In the case of two MF$\mathrm{s}\left(\gamma_{1}, \gamma_{2}\right)$, the two MFs may be combined into a single complex fermion as $d=\left(\gamma_{1}+i \gamma_{2}\right) / 2, d^{\dagger}=\left(\gamma_{1}-i \gamma_{2}\right) / 2$. The ground state is doubly degenerate. To label the two-fold degenerate ground state, we introduce the Fermion-parity operator $\hat{\mathrm{P}}=\left(-i \gamma_{1} \gamma_{2}\right)$. Because $\hat{\mathrm{P}}^{2}=1, \hat{\mathrm{P}}$ has two eigenvalues \pm 1 , which corresponds even or odd Fermion-parity ground state. With the help of $d$ and $d^{\dagger}$, two Majorana modes $\left(\gamma_{1}, \gamma_{2}\right)$ form a physical Fermion-parity qubit: $|e\rangle$ is a many-body quantum state with even Fermion-parity, and $d^{\dagger}|e\rangle=|o\rangle$ is a many-body quantum state with odd Fermion-parity. Generally, there is coupling between two MFs, and the effective Hamiltonian is given by

$$
i \mathcal{J} \gamma_{1} \gamma_{2}=2 \mathcal{J}\left(d^{\dagger} d-\frac{1}{2}\right)
$$

where $\mathcal{J}$ is the coupling constant. Therefore, the two Majorana zero modes evolve into a pair of levels $(\mathcal{J},-\mathcal{J})$ with a splitting energy $2 \mathcal{J}$.

To distinguish the statistics of the MFs, we perform the braiding operation on MFs by adiabatically deforming the system, for example, changing the length of a 1D nano-wire or moving the vortices. In other words, the braiding operations for MFs are really adiabatic evolutions of the system. According to the 
results in Ref.[25], if we exchange two MFs with non-local $\pi$ phase strings, then the resulting braiding operation is given by

$$
\gamma_{i} \rightarrow \gamma_{j}, \gamma_{j} \rightarrow-\gamma_{i}
$$

that can be described by an "unitary" transformation $U_{i j}=$ $e^{\frac{\pi}{4} \gamma_{j} \gamma_{i}}$ [25]. For three MFs $\gamma_{i}, \gamma_{j}$, and $\gamma_{k}$, as $U_{i j}$ and $U_{j k}$ do not commute, i.e. $\left[U_{i j}, U_{j k}\right] \neq 0$, the exchange statistics of MFs is non-Abelian and belongs to the Ising anyon class. Based on braiding operations on Ising anyons that obey non-Abelian statistics, the Hadamard gate, the phase gate, and the CNOT gate can be treated topologically, except for the $\pi / 8$ gate[30, 31, 23]. In the case of two MFs, the braiding operator is represented by $U_{12}=e^{\frac{\pi}{4} \gamma_{2} \gamma_{1}}=e^{i \frac{\pi}{4}\left(2 d^{\dagger} d-1\right)}=e^{i \pi \sigma^{z} / 4}$, where $\sigma^{z}$ is a Pauli matrix in the basis of two nearly degenerate ground states $\left(d^{\dagger}|e\rangle,|e\rangle\right)$. Thus, the relative phase between the two nearly degenerate ground states obtained from the braiding is $\pi / 2$.

Based on two typical topological SCs, 1D $p$-wave topological SC and 2D $p_{x}+i p_{y}$-wave topological SC, we verify the non-Abelian statistics of emergent MFs in SCs by simulating the braiding processes in BdG representation numerically. To characterize the braiding process, we introduce two parameters: the amplitude of the BdG function-overlap $O=\left|\left\langle\psi_{\mathrm{i}} \mid \psi_{\mathrm{f}}\right\rangle\right|$ and the relative Berry phase $\Phi .\left|\psi_{\mathrm{i}}\right\rangle$ is the initial BdG wave function with Majorana modes at two ends of the defect-line or $\pi$ phase string, and $\left|\psi_{\mathrm{f}}\right\rangle$ is the $\mathrm{BdG}$ wave function evolution from $\left|\psi_{\mathrm{i}}\right\rangle$ after braiding processes. The results $O=1$ and $\Phi=\pi / 2$ after the braiding process suggest MFs obey non-Abelian statistics. Therefore, we can distinguish the statistics for the MFs by calculating $O$ and $\Phi$.

\section{Results}

The first model is 1D $p$-wave SC on T-junction, as illustrated in Fig.1.(a), which consists of two parts, a "-"-line $(A \leftrightarrow C$, of which the length is $L_{-}$) and a "|"-line ( $\mathrm{D} \leftrightarrow \mathrm{B}$ of which the length is $L_{\mid}$). The pair order parameter on "-"-line is $\Delta$ and that on "|"-line is $i \Delta$. The Hamiltonian of the system can be written as $H=H_{-}+H_{\mid}+H_{D}$, where

$$
\begin{aligned}
& H_{-}=-J \sum_{j=1}^{L_{-}-1} c_{j+1}^{\dagger} c_{j}+\Delta \sum_{j=1}^{L_{-}-1} c_{j+1}^{\dagger} c_{j}^{\dagger}+h . c .-\sum_{j=1}^{L_{-}} \mu_{j} c_{j}^{\dagger} c_{j}, \\
& H_{\mid}=-J \sum_{j=1}^{L_{1}-1} a_{j+1}^{\dagger} a_{j}+i \Delta \sum_{j=1}^{L_{\mid}-1} a_{j+1}^{\dagger} a_{j}^{\dagger}+h . c-\sum_{j=1}^{L_{\mid}} \mu_{j} a_{j}^{\dagger} a_{j}, \\
& H_{D}=J a_{j=1}^{\dagger} c_{j_{D}}+i \Delta a_{j=1}^{\dagger} c_{j_{D}}^{\dagger}+\text { h.c. }
\end{aligned}
$$

where $c_{j}\left(a_{j}\right)$ is the annihilation operator of spinless fermions on the "- $(\mid)$ "-line, and $j_{D}$ denotes the touching point of "|"-line on "-"-line (D point in Fig.1.(a)). $J$ is the hopping strength, $\Delta$ is the superconducting pairing order parameter, and $\mu_{j}$ is the on-site chemical potential. In the following, we choose $\Delta=J$, and the lattice constant is set to be unity.

For the case of $|\mu|<2 J$, the ground state is a topological SC; for the case of $|\mu|>2 J$, the ground state is a non-topological SC. In Fig.1.(a), we denote the topological $p$-wave SC by the

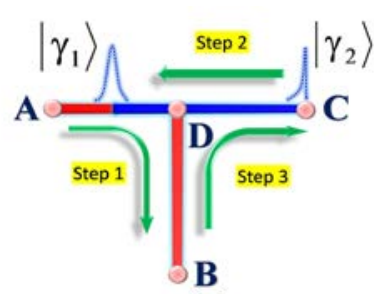

(a)
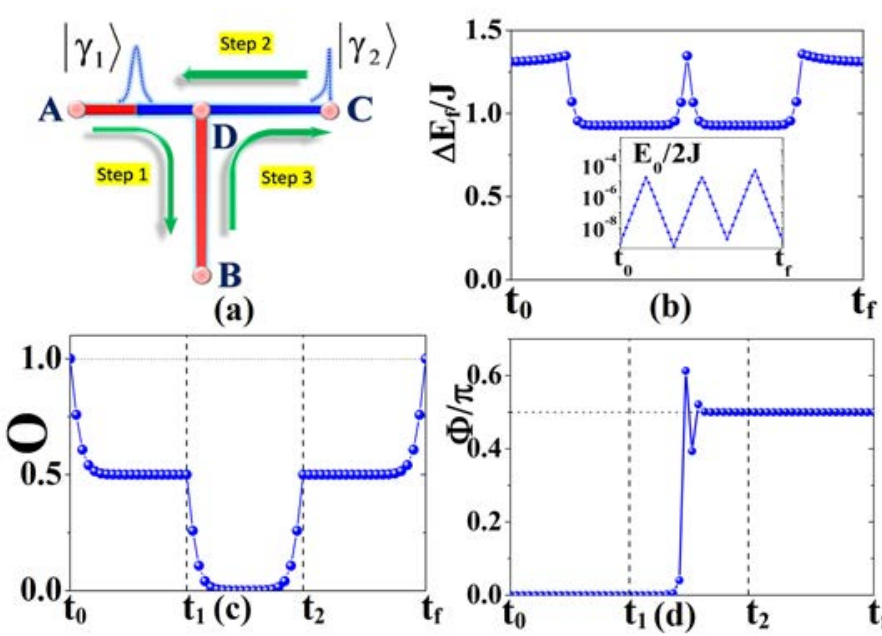

(b)

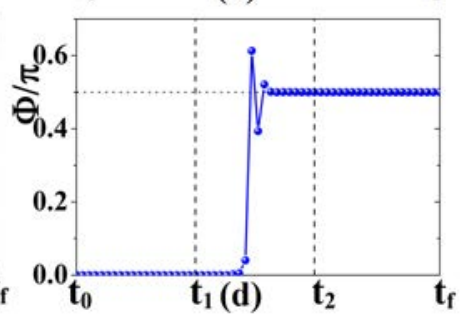

Figure 1: (color online) (a) Illustration of the T-junction and T-shape braiding path. We denote the topological $p$-wave SC by the blue line and the nontopological $p$-wave SC by the red line. As a result, there always exists a pair of MFs $\left(\gamma_{1}, \gamma_{2}\right)$ at two ends of the blue lines. (b) The energy gap of the system, $\Delta E_{f}$. The inset is the energy $E_{0}$ of the $\mathrm{BdG}$ state with the Majorana modes at the two ends. (c) The function-overlap $O=\left|\left\langle\psi_{\mathrm{i}} \mid \psi_{\mathrm{f}}\right\rangle\right|$ of the Majorana modes during the adiabatic evolutions (the dotted line denotes 1). (d) The relative Berry phase $\Phi$ (the dotted lines denotes $\pi / 2$ ). In all these figures, we choosing the parameters as $\Delta=J$.

blue line and non-topological $p$-wave SC by the red line. As a result, there always exists a pair of MFs $\left(\gamma_{1}, \gamma_{2}\right)$ at two ends of the topological $p$-wave SC (a blue line). For the initial state $\left(\mathrm{t}=\mathrm{t}_{0}\right)$, we set $\mu=-0.7 \mathrm{~J}$ on the "-"-line and $\mu=-10 \mathrm{~J}$ on the "|"-line. Thus, the $p$-wave SC on the "-"-line is topological, whereas the $p$-wave SC on "l"-line is non-topological. By numerical calculations on the T-junction with $L_{-}=20, L_{\mid}=10$, we determine that two MBS BdG functions have distributions of two MFs of $\left|\gamma_{1}\right\rangle$, and $\left|\gamma_{2}\right\rangle$ near A and C, respectively.

In the following parts, we will show how to braid the two MFs $\left(\gamma_{1}, \gamma_{2}\right)$ at two ends of "-"-line. As shown in Fig.1.(a), we can braid two MFs $\left(\gamma_{1}, \gamma_{2}\right)$ through site-by-site tuning of the chemical potential from $\mu=-0.7 J$ to $\mu=-10 J$ or from $\mu=-10 \mathrm{~J}$ to $\mu=-0.7 \mathrm{~J}$ in a three-step process: step 1 involves moving MF $\gamma_{1}$ from $\mathrm{A}$ to $\mathrm{B}$ through $\mathrm{D}$ as $\mathrm{A} \rightarrow \mathrm{D} \rightarrow \mathrm{B}$ during the time period $t_{1}-t_{0}$; step 2 involves moving $\gamma_{2}$ from $C$ to $A$ through $\mathrm{D}$ during the time period $\mathrm{t}_{2}-\mathrm{t}_{1}$; step 3 involves moving $\gamma_{1}$ from $\mathrm{B}$ to $\mathrm{C}$ through $\mathrm{D}$ during the time period $\mathrm{t}_{f}-\mathrm{t}_{2}$.

To guarantee the adiabatic condition, the time period $\Delta \mathrm{t}$ for moving an MF one lattice constant should be very large, i.e., $\Delta \mathrm{t} \gg \hbar / \Delta E_{f}$. In this experiment, we choose $\Delta \mathrm{t}=10000 \hbar / J$. The total time period for the braiding operation is $\mathrm{t}_{f}-\mathrm{t}_{0}=58 \Delta \mathrm{t}$, and the time periods for the three steps are $\mathrm{t}_{1}-\mathrm{t}_{0}=\mathrm{t}_{2}-\mathrm{t}_{1}=$ $19 \Delta \mathrm{t}, \mathrm{t}_{f}-\mathrm{t}_{2}=20 \Delta \mathrm{t}$, respectively. During the braiding process, as shown in Fig.1.(b), the Hamiltonian remains gapped at any time in which the minimum value of energy gap $\Delta E_{f}$ is approximately $0.9 \mathrm{~J}$, which protects the topological properties of the system and the stability of the MFs, and in which the maximum value of the splitting energy $E_{0}$ due to the tunneling between the two MFs is approximately $10^{-5} \mathrm{~J}$, which leads to a small dynamical phase. After calculating $\mathcal{T}\left\{\exp \left[i \int_{t_{0}}^{t_{f}} H_{B d G}(t) d t\right]\left|\psi_{\mathrm{i}}\right\rangle\right\}$, 
we derive the dynamical phase and Berry phase of the Majorana zero modes during the braiding process. Here, $\mathcal{T}$ is the time-ordered-product operator.

The final results are given in Fig.1.(c) and Fig.1.(d). For the initial state $\left|\psi_{\mathrm{i}}\right\rangle$, after the braiding operation, we obtain the Berry phase $\Phi=\pi / 2$. Fig.1.(d) shows that the Berry phase $\Phi$ changes abruptly during MF $\gamma_{2}$ crossing D point. The fidelity $\left|\left\langle\psi_{\mathrm{i}} \mid \psi_{\mathrm{f}}\right\rangle\right|$ is very close to $100 \%$. As a result, we verify the non-Abelian-statistics of MFs in the 1D p-wave topological SC system.

The second model is $p_{x}+i p_{y}$-wave SC, for which the Hamiltonian can be written as[24]

$$
\begin{aligned}
H & =-\sum_{j} \sum_{\widehat{\mu}=\widehat{x, y}} J_{j}\left(c_{j+\widehat{\mu}}^{\dagger} c_{j}+c_{j-\widehat{\mu}}^{\dagger} c_{j}\right) / 2-\mu \sum_{j} c_{j}^{\dagger} c_{j} \\
& +\sum_{j}\left[\Delta_{j}\left(c_{j+\widehat{x}}^{\dagger} c_{j}^{\dagger}+i c_{j+\widehat{y}}^{\dagger} c_{j}^{\dagger}\right) / 2+\text { h.c. }\right],
\end{aligned}
$$

where $c_{j}^{\dagger} / c_{j}$ is the creation/annihilation operator of a spinless fermion. $J_{j}$ is the hopping strength, $\Delta_{j}$ is the superconducting pairing order parameter, and $\mu$ is the chemical potential. In the following, we choose the parameters of $\Delta_{j}=J_{j}=J, \mu=J$, for which the ground state is a topological SC with non-zero Chern number. The lattice constant is also set to be unity.

In the topological phase of $p_{x}+i p_{y}$-wave SC, we study two $\operatorname{MFs}\left(\gamma_{1}, \gamma_{2}\right)$ around two quantized vortices ( $\pi$-fluxes) by numerical calculations on a $20 \times 20$ lattice. The dotted line denotes the phase branch-cut of the two $\pi$-fluxes (denoted here as A-B$\mathrm{C} \pi$-phase string), along which the hopping parameters and the pairing order parameters change signs, $J_{j} \rightarrow-J_{j}, \Delta_{j} \rightarrow-\Delta_{j}$. Thus, there exists a Majorana zero mode around each end of the $\pi$-phase strings. See the particle density distribution of Majorana modes $\left(\left|\gamma_{1}\right\rangle,\left|\gamma_{2}\right\rangle\right)$ around $\pi$-fluxes in Fig.2.(a).

To braid the two MFs $\left(\gamma_{1}, \gamma_{2}\right)$, we choose a $\square$-shape path rather than a traditional T-shape path. As shown in Fig.2.(b), we move in the anticlockwise direction two MFs $\left(\gamma_{1}, \gamma_{2}\right)$ by moving the $\pi$-phase string through a two-step process: step 1 involves moving MF $\gamma_{1}$ from A to B together with moving MF $\gamma_{2}$ from $\mathrm{C}$ to $\mathrm{D}$ during the time period $\mathrm{t}_{1}-\mathrm{t}_{0}$; step 2 involves moving MF $\gamma_{1}$ from $\mathrm{B}$ to $\mathrm{C}$ together with moving MF $\gamma_{2}$ from $\mathrm{D}$ to A during the time period $t_{f}-t_{1}$. However, after the two-step braiding process, the A-B-C $\pi$-phase string changes into a C-DA $\pi$-phase string. Thus, to return the initial configuration, we must deform the C-D-A $\pi$-phase string to A-B-C $\pi$-phase string by performing local $\mathrm{Z} 2$ transformation on the fermion fields inside the $\square$-shape closed loop A-B-C-D, $c_{j} \rightarrow-c_{j}$. Eventually, we adiabatically perform the braiding operation.

Next, we derive the Berry phases of BdG states $\left|\psi_{\mathrm{i}}\right\rangle$ by calculating $\left.\mathcal{T}\left\{\exp \left[i \int_{t_{0}}^{t_{f}} H(t) d t\right]\left|\psi_{\mathrm{i}}\right\rangle\right)\right\}$. In numerical calculation$\mathrm{s}$, the time period for moving an MF one lattice constant is $\Delta \mathrm{t}=400 \hbar / J$ (or the total time period for the braiding operation is $\mathrm{t}_{f}-\mathrm{t}_{0}=20 \Delta \mathrm{t}$ and $\mathrm{t}_{1}-\mathrm{t}_{0}=\mathrm{t}_{f}-\mathrm{t}_{1}=10 \Delta \mathrm{t}$ ). During the braiding operation, the energy gap of the system is always $\Delta E_{f} \equiv 2 J$, and the energy splitting between two BdG states of Majorana modes is extremely small, $\max \mathcal{J}<10^{-7} \mathrm{~J}$. As a result, the dynamical phases of the BdG state $\left|\psi_{\mathrm{i}}\right\rangle$ are approxi-

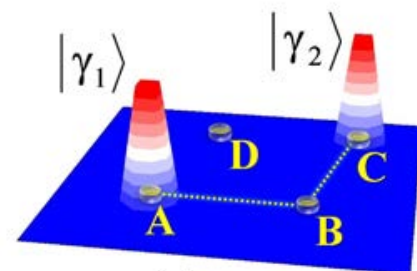

(a)
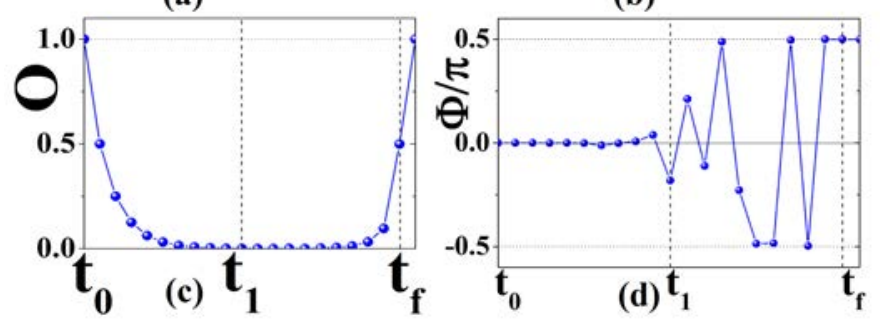

Figure 2: (color online) (a) The particle density distribution of Majorana modes $\left(\left|\gamma_{1}\right\rangle,\left|\gamma_{2}\right\rangle\right)$ around two $\pi$-fluxes in a $p_{x}+i p_{y}$ topological superconductor. The dotted line denotes the A-B-C $\pi$-phase string that connects the two MFs. (b) The $\square$-shape path of the two-step braiding process: $\mathrm{I} \rightarrow \mathrm{II}\left(\mathrm{t}_{0}<\mathrm{t}<\mathrm{t}_{1}\right)$ and $\mathrm{II} \rightarrow \mathrm{III}\left(\mathrm{t}_{1}<\mathrm{t}<\mathrm{t}_{f}\right)$. III $\rightarrow$ IV denotes the string deformation. (c) The functionoverlap $O=\left|\left\langle\psi_{\mathrm{i}} \mid \psi_{\mathrm{f}}\right\rangle\right|$ of the Majorana modes during the adiabatic evolutions (the dotted line denotes 1 ). (d) The relative Berry phase $\Phi$ (the dotted lines denote $\pm \pi / 2$ ). In all these figures, we choose the parameters as $\Delta=J, \mu=J$. The last spots of the data in (c) and (d) are obtained from string deformation rather than braiding operations.

mately $\pm 10^{-5} \pi$, i.e., too small to cause errors in the Berry phase calculations.

The results of the amplitude of the function-overlap $O=$ $\left|\left\langle\psi_{\mathrm{i}} \mid \psi_{\mathrm{f}}\right\rangle\right|$ and the Berry phase $\Phi$ are given in Fig.2.(c) and Fig.2.(d), respectively. Fig.2.(d) shows that the relative Berry phase is approximately $\Phi=0.49987 \pi$, which is quite close to $\pi / 2$. The fidelity $O=\left|\left\langle\psi_{\mathrm{i}} \mid \psi_{\mathrm{f}}\right\rangle\right|$ is up to $99.98 \%$. As a result, MFs induced by the vortices in a $p_{x}+i p_{y}$ topological SC obey non-Abelian statistics.

\section{Conclusions}

In this paper, we developed a numerical method from the relationship between the braiding operator of MFs and that of B$\mathrm{dG}$ states and verified non-Abelian statistics of MFs in 1D and 2D topological SCs using this numerical method. The numerical results confirmed our prediction exactly.

In the end, we addressed the physical realization of the above braiding process in a cold atom system. The effective $p_{x}+i p_{y}$ superfluid state in the optical lattice has been proposed to be realized through the p-wave Feshbach resonance[32] or s-wave Feshbach resonance making use of a topological Berry phase[33]. However, due to considerable progress in addressability of a single atom in optical lattice, the in-situ manipulation of optical lattice can be achieved in experiments[34, 35, $36,37]$. In the future, the braiding of Majorana fermions may be realized through using optical tweezers[38] and has potential to be applied in topological quantum computation. 


\section{Acknowledgements}

This work is supported by National Basic Research Program of China (973 Program) under the grant No. 2011CB921803, 2012CB921704 and NSFC Grant No.11174035, 11474025, 11404090, SRFDP, the Fundamental Research Funds for the Central Universities, NSF-Hebei Province under Grant No. A2015205189 and NSF-Hebei Education Department under Grant No. QN2014022.

\section{References}

[1] E. Majorana, Soryushiron Kenkyu 63, 149 (1981).

[2] F. Wilczek, Nature Phys. 5, 614 (2009).

[3] M. Leijnse and K. Flensberg, Semicond. Sci. Technol. 27, 124003 (2012).

[4] A. Y. Kitaev, Phys. Usp. 44, 131 (2001).

[5] L. Fu and C. L. Kane, Phys. Rev. Lett. 100, 096407 (2008).

[6] R. M. Lutchyn, et.al, Phys. Rev. Lett. 105, 077001 (2010).

[7] Y. Oreg, et.al, Phys. Rev. Lett. 105, 177002 (2010).

[8] J. D. Sau, et.al, Phys. Rev. Lett. 104, 040502 (2010).

[9] J. Alicea, Phys. Rev. B 81, 125318 (2010).

[10] A. C. Potter and P. A. Lee, Phys. Rev. Lett. 105, 227003 (2010).

[11] J. Alicea, et.al, Nature Phys. 7, 412 (2011).

[12] B. I. Halperin, et.al, Phys. Rev. B 85, 144501 (2012).

[13] T. D. Stanescu and S. Tewari, J. Phys. C 25, 233201 (2013).

[14] V. Mourik, et.al, Science 336, 1003 (2012).

[15] A. Das, et.al, Nature Phys. 8, 887 (2012).

[16] M. T. Deng, et.al, Nano Lett. 12, 6414 (2012).

[17] L. P. Rokhinson, et.al, Nat. Phys. 8, 795 (2012).

[18] H. O. H. Churchill, et.al, Phys. Rev. B 87, 241401(R) (2013).

[19] A. Kitaev, Ann. Phys. 321, 2 (2006).

[20] C. Nayak, et.al, Rev. Mod. Phys. 80, 1083 (2008).

[21] M. H. Freedman, et.al, Math. Phys. 227, 605 (2002).

[22] S. Das Sarma, et.al, Phys. Rev. Lett. 94, 166802 (2005).

[23] L. S. Georgiev, Phys. Rev. B 74, 235112 (2006); L. S. Georgiev, Nucl. Phys. B 789, 552 (2008).

[24] N. Read and D. Green, Phys. Rev. B 61, 10267 (2000).

[25] D. A. Ivanov, Phys. Rev. Lett. 86, 268 (2001).

[26] J. Alicea, Rep. Prog. Phys. 75, 076501 (2012).

[27] Y. J. Wu, et.al, Phys. Rev. A. 90, 022324 (2014).

[28] G. Moore and N. Read, Nucl. Phys. B 360, 362 (1991).

[29] Xiao-Gang Wen, Phys. Rev. Lett. 66, 802 (1991).

[30] C. Nayak, \& F. Wilczek, Nucl. Phys. B 479, 529-553 (1996).

[31] M. Freedman, et.al, Phys. Rev. B 73, 245307 (2006).

[32] V. Gurarie, L. Radzihovsky, a. V. Andreev, Phys. Rev. Lett. 94, 230403 (2005).

[33] C. Zhang, S. Tewari, R.M. Lutchyn, S. Das Sarma, Phys. Rev. Lett. 101, 160401 (2008).

[34] P. Wurtz, T. Langen, T. Gericke, A. Koglbauer, H. Ott, Phys. Rev. Lett. 103, 080404 (2009).

[35] N. Gemelke, X. Zhang, C.-L. Hung, C. Chin, Nature. 460, 995 (2009).

[36] J.F. Sherson, C. Weitenberg, M. Endres, M. Cheneau, I. Bloch, S. Kuhr, Nature. 467, 68 (2010).

[37] C. Weitenberg, et al., Nature. 471, 319 (2011).

[38] S. Tewari, S. Das Sarma, C. Nayak, C. Zhang, P. Zoller, Phys. Rev. Lett. 98, 010506 (2007). 